\title{
Assessment of the Machine Dynamic Quality on the Basis of the Integrated Assessment of the Spectrum of Vibroacoustic Vibrations
}

\author{
Shamsadova Ya.Sh. \\ Professor of Institute of Oil and Gas \\ Grozny State Oil Technical University \\ Grozny, Russia \\ turluevadam@mail.ru
}

Ignatiev A.A.

Doctor of Technical Sciences,

Professor "Automation, control, mechatronics"

Saratov State Technical University

Saratov, Russia

atp@sstu.ru

Dobryakov V.A.

Ph.D. Associate Professor "Automation, control, mechatronics"

Saratov State Technical University

Saratov, Russia

atp@sstu.ru

Sadykov Kh.A.

Associate Professor Institute of Oil and Gas

Grozny State Oil Technical University

Grozny, Russia

has.sattoy@mail.ru

\author{
Mintsaev M.S. \\ Doctor of Technical Sciences, \\ Professor Institute of Oil and Gas \\ Grozny State Oil Technical University \\ Grozny, Russia \\ ranas@rambler.ruu
}

Ignatiev S.A.

Doctor of Technical Sciences, Professor "Automation, control, mechatronics"

Saratov State Technical University

Saratov, Russia

atp@sstu.ru

Pashaev M.Y.

Ph.D. Professor, Senior Lecturer Institute of Oil and Gas

Grozny State Oil Technical University

Grozny, Russia

south-glonass@mail.ru

Sadykova Z.V.

Professor Institute of Oil and Gas

Grozny State Oil Technical University

Grozny, Russia

srfggy@mail.ru

\begin{abstract}
Dynamic quality of machines is one of the dominant factors affecting both the geometric parameters of the accuracy of components, and the physicomechanical characteristics of the treated surface layer. Individually, each machine has a certain dynamic quality, which varies both in the process of operation and when the values of cutting mode parameters are changed. What is important in the manufacture of precision machine- and instrument components making is the assessment of the actual dynamic quality of machines according to the characteristics of vibroacoustic vibrations.
\end{abstract}

Keywords - grinding machine, dynamic quality, vibroacoustic vibrations, spectral density, autocorrelation, integrated assessment, bearing rings, surface waviness.

\section{INTRODUCTION}

The quality of products in machine building and instrument making largely depends on both the geometric accuracy of the parts manufactured and the physicomechanical characteristics of the treated surface layer, which significantly affects the reliability of products in operation $[1,2]$.

One of the dominant factors affecting the quality of machining parts on automated metal-cutting machine tools (MCT) is their dynamic quality, which is ensured at the design and manufacture stages of machine tools and is maintained at the operation stage $[3,4]$. The dynamic quality of machines is estimated based on the measurement of vibroacoustic (VA) oscillations, therefore, an important point for production conditions is to determine the dynamic quality of each machine (if similar parts are processed and similar cutting conditions are used). Moreover, it is important to determine the values of cutting mode parameters for each machine, at which the level of VA oscillation of the main components involved in the shaping process is minimal, so that low quality processing is reached [4-6]. 
When machining bearing rings on grinding machines of various models, various estimates of the VA dynamic system were obtained. Firstly, the dynamic quality of the machine was evaluated. Secondly, the values of cutting mode parameters were corrected to obtain a specified machining accuracy $[4,7$, 8]. As integral estimations of spectral densities and integral estimates of autocorrelation functions (ACF) of oscillations were used, as well as the stability margin of the dynamic system (DS) of the machine. The objective of this paper is to state that the regularity of the measurement of integral estimations of the spectral density of VA dynamic system is similar to both the change in the integral estimations of the ACF and the stability margin of the DS, so that they can be used equally to assess the machine dynamic quality.

\section{METHODS AND MATERIALS}

One of the methods for assessing the dynamic quality of turning and grinding machines is based on the determination of ACF VA oscillations, and uses two options for further processing ACF $\mathrm{K}(\tau)$ with the help of specialized programming mathematical software (PMS).

The first option is connected with the formation of integral assessments of the ACF in the form of [10]

$$
I_{A}=\int_{o}^{\tau_{K}} K^{2}(\tau) d \tau
$$

$\tau_{\mathrm{K}}-$ correlation time.

This option has been tested to select the appropriate speed of rotation of the workpiece when machining on a precision turning module such as TPARM. Measurement of vibrations in the frequency band up to $4 \mathrm{kHz}$ was carried out using a vibration meter VSHV-003M2, the DN-3 sensor of which was fixed on a magnetic support on the cutter block $[9,10]$. At the minimum of the integral estimation (1), the spindle rotation frequency was assigned, at which the roughness parameters of the machined surface $\mathrm{Ra}$ (about $0.1 \mu \mathrm{m}$ ) and the machining time of the component were minimal.

The second use of the ACF is based on the calculation of the transfer function of the DS during cutting, and it is assumed that the cutting force forced by the DS is a "white noise" signal [11, 12]. Based on this, it was found in [10] that the oscillation index $\mathrm{M}$ calculated from the transfer function, on the basis of which the stability margin of the DW is estimated, changes similarly with the change in the integral estimation IA, and can also be used to select the cutting mode.

To assess the dynamic quality of the turning machine in different cutting conditions, the use of an integral estimation of the spectral density S $(\omega)$ VA of the DC was tested.

$$
I_{A}=\int_{\omega_{1}}^{\omega_{2}} S(\omega) d u,
$$

$\omega_{1}, \omega_{2}-$ lower $\omega_{1}$ and upper bounds of the frequency range of measured oscillations VA.

The results of experimental studies on a turning module of the TPARM type, shown in Fig. 1, show that the minimum value of the integrated assessment of the IC spectrum corresponds to the minimum surface roughness $\mathrm{Ra}$ at $\mathrm{n}=1600$ rpm, and the minimum value of the oscillation index corresponds to the maximum stability margin of the DS. It correlates with the data of [10].

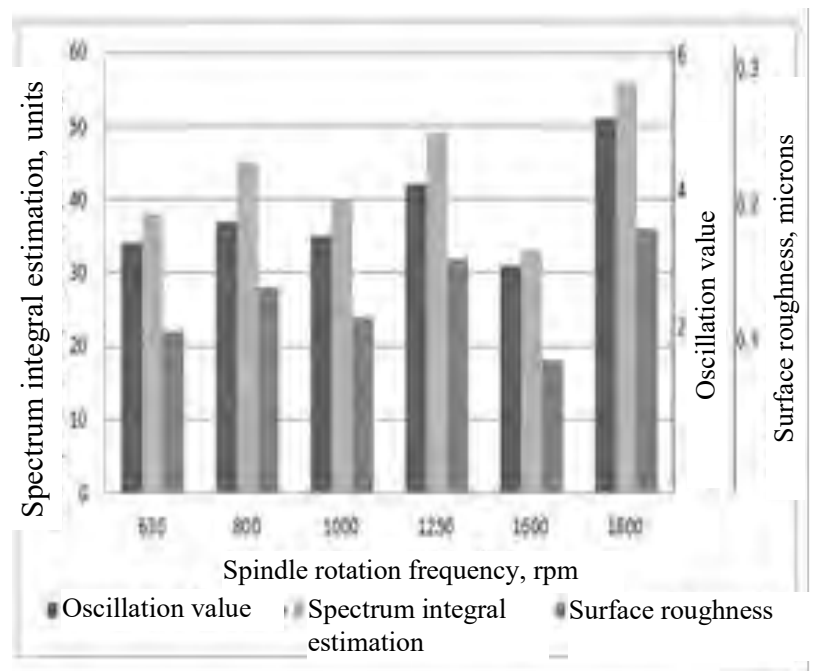

Fig. 1. Changing the integral estimates of the spectrum, the index of oscillation and surface roughness when machining on the TPARM turning module

Next, we examine the use of integral estimates on grinding machines for machining the raceways of bearing rings. In [7], it was found that the integral estimates of the spectral density $\mathrm{S}$ () of the VA oscillations can be used to assess the dynamic quality of the SWaAGL-50 grinding machines. From the results of internal grinding machines of the SiN-5 model studies, it can be seen (Fig. 2) that the value of the quality parameter "waviness" of the raceway is minimal for the machine No. 395, which has the lowest values of the integral estimates of the Ic spectrum.

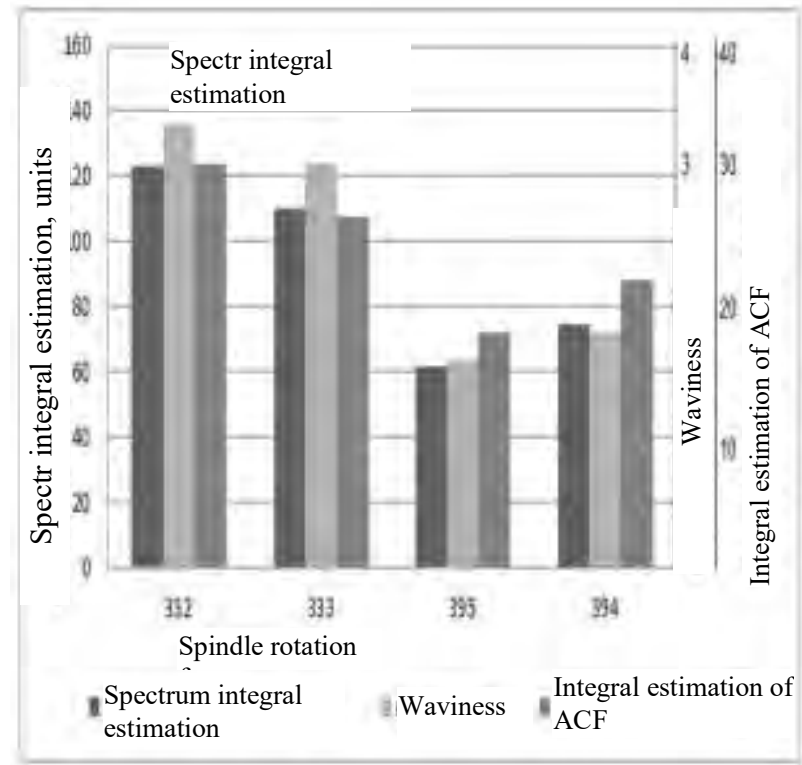

Fig. 2. The value of the integral spectrum estimates of the oscillation index of the internal grinding machines $\mathrm{SiN}-5$ and the waviness of the raceways bearing rings (average value over 5 rings, acceptable value of $2 \mu \mathrm{m}$ )

In the experiments on grinding machines, as well as for turning machines, a vibration meter VSHV-003M2 with a DN3 sensor was used, which was mounted on a ring support near 
the cutting zone. The signal from the vibro-meter was processed on a computer by specialized software that allows to calculate the ACF $\mathrm{K}(\tau)$, the spectrum $\mathrm{S}(\omega)$ and their integral estimates.

In the works $[2,4]$ it is indicated that for grinding machines experimental ACF can be approximated by the formula

$$
K(\tau)=K_{o} e^{-\propto \tau} \cos \omega_{o} \tau,
$$

$K_{\mathrm{o}}-\mathrm{ACF}$ value at $\tau=0$, which without loss of generality can be taken $K_{o}=1 ; \alpha$-attenuation coefficient, $\omega_{O}$ - frequency of damped oscillations ACF.

The integral estimates $I_{A}$ and $I_{c}$ have similar patterns of change with increasing $\alpha$.

Spectral density (or spectrum) $\mathrm{S}(\omega)$ is connected with ACF known ratio

$$
S(\omega)=\int_{-\infty}^{\infty} K(\tau) e^{-j \omega \tau} d \tau
$$

Due to the fact that the ACF is an even function [13] follows

$$
S(\omega)=2 \int_{0}^{\infty} K(\tau) \cos \omega \tau d ? .
$$

By substituting it in (5) formula for ACF (3) with $K_{0}=1$ we get

$$
S(\omega)=2 \int_{0}^{\infty} e^{-l \tau} \cos \left(\omega_{o} \tau\right) \cos \omega \tau d \tau
$$

Integral calculation leads to the following formula

$$
S(\omega)=\frac{2 \alpha\left(\alpha^{2}+\omega^{2}+\omega_{0}^{2}\right)}{\left[\alpha^{2}+\left(\omega-\omega_{0}\right)^{2}\right]\left[\alpha^{2}+\left(\omega+\omega_{0}\right)^{2}\right]}
$$

It is rather difficult to calculate the integral estimate Ic from formula (7), therefore, we decompose its right side into two summands using the method of undetermined coefficients:

$$
\begin{gathered}
\frac{\alpha\left(\alpha^{2}+\omega^{2}+\omega_{0}^{2}\right)}{\left[\alpha^{2}+\left(\omega-\omega_{0}\right)^{2}\right]\left[\alpha^{2}+\left(\omega+\omega_{0}\right)^{2}\right]}=\frac{A \alpha+B}{\alpha^{2}+\left(\omega-\omega_{0}\right)^{2}} \\
\frac{C \alpha+D}{\alpha^{2}+\left(\omega-\omega_{0}\right)^{2}}
\end{gathered}
$$

The numerator of the right side of $\mathrm{H}(\alpha)$ after reduction to a common denominator is

$$
\begin{array}{r}
\mathrm{H}(\alpha)=A \alpha^{3}+A \alpha \omega_{-}^{2}+B \alpha^{2}+B \omega_{-}^{2}+C \alpha^{3}+C \alpha \omega_{+}^{2}+ \\
D \alpha^{2}+D \omega_{+}^{2},
\end{array}
$$

где $\omega_{-}=\omega-\omega_{0}, \omega_{+}=\omega+\omega_{0}$

Comparing the coefficients of the numerator of the left side of equation (8) with the coefficients for the corresponding powers of $\alpha$ in formula (9) we have:

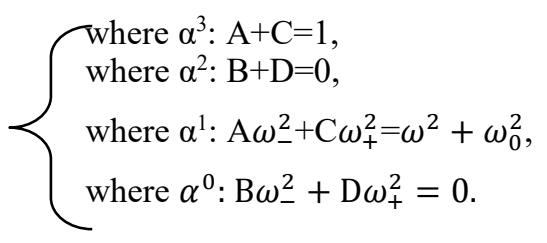

From four equations (10) we obtain the values of the coefficients

$$
\mathrm{A}=\mathrm{C}=0,5 ; \mathrm{B}=\mathrm{D}=0
$$

Formula (7) in this case is transformed:

$$
S(\omega)=\frac{\alpha}{\alpha^{2}+\left(\omega+\omega_{0}^{2}\right)^{2}}+\frac{\alpha}{\alpha^{2}+\left(\omega-\omega_{0}\right)^{2}} .
$$

Integral estimate of the first summand $\mathrm{I}_{\mathrm{c} 1}(\alpha)$ of formula (11) is the following:

$$
\mathrm{I}_{\mathrm{c} 1}(\alpha)=\alpha \int_{\omega_{1}}^{\omega_{2}} \frac{d \omega}{\alpha^{2}+\left(\omega+\omega_{0}\right)^{2}}=\left.\operatorname{arctg}\left(\frac{\omega+\omega_{0}}{\alpha}\right)\right|_{\omega_{1}} ^{\omega_{2}}
$$

Integral estimate of the second summond $\mathrm{I}_{\mathrm{c} 2}(\alpha)$ of formula (11) is the following:

$$
\mathrm{I}_{\mathrm{c} 2}(\alpha)=\alpha \int_{\omega_{1}}^{\omega_{2}} \frac{d \omega}{\alpha^{2}+\left(\omega-\omega_{0}\right)^{2}}=\left.\operatorname{arctg}\left(\frac{\omega-\omega_{0}}{\alpha}\right)\right|_{\omega_{1}} ^{\omega_{2}}
$$

To identify trends in the integral estimation $\mathrm{I}_{\mathrm{c} 1}(\alpha)$ with increasing attenuation coefficient $\alpha$, we calculate the derivative with respect to $\alpha$ and determine its sign. Taking into account formulas (12) and (13) we have:

$$
\begin{array}{r}
\frac{d I_{C}(\alpha)}{d \alpha}=\frac{d I_{C 1}(\alpha)}{d \alpha}+\frac{d I_{C 2}(\alpha)}{d \alpha}=-\frac{\omega_{2}+\omega_{0}}{\alpha^{2}+\left(\omega_{2}+\omega_{0}^{2}\right)^{2}}+ \\
\frac{\omega_{1}+\omega_{0}}{\alpha^{2}+\left(\omega_{1}+\omega_{0}\right)^{2}}-\frac{\omega_{2}-\omega_{0}}{\alpha^{2}+\left(\omega_{2}-\omega_{0}^{2}\right)^{2}}+\frac{\omega_{1}-\omega_{0}}{\alpha^{2}+\left(\omega_{1}+\omega_{0}\right)^{2}} .
\end{array}
$$

The first and third terms in (14) are less than zero. After algebraic transformations, it can be shown that the sum of the second and fourth summonds is also less than zero, given that $\omega_{1}<\omega_{0}<\omega_{2}$. Therefore, $d \mathrm{I}_{\mathrm{c}}(\alpha) / d \alpha<0$, that is, the integral estimate of the spectrum is monotonously decreasing, as is the integral estimate of the ACF and the oscillation index.

Experimental studies of VA oscillations performed on the internal grinding machines of the SIW-5 model during the processing the bearing rings raceways confirmed that the change in the integral spectrum estimates for different machines change in a similar way to the change in the integral ACF estimates. Comparison of these estimates with processing accuracy indices shows that machine No. 395, which has the minimum values for the studied machines, also provides higher processing accuracy - the smallest values of the ovality and waviness of the raceway.

Thus, the considered estimates can serve as an assessment of the dynamic quality of machine tools.

\section{CONCLUSION}

The results of theoretical and automated experimental studies of vibro-acoustic oscillations of machine tools and the accuracy of machining showed that the assessment of the dynamic quality of machines according to integral estimates of the spectral density of oscillations correlates with the integral estimates of the ACF and the oscillation index determining the stability margin of the dynamic system of the machine.

Thus, the analysis of the vibroacoustic characteristics of the machines with the use of these three estimates allows to rank the machines according to dynamic quality and machining accuracy; to determine the values of cutting mode parameters with the highest machining accuracy; to give recommendations on carrying out restoration work on machines that do not meet the specified values of machining accuracy.

\section{References}

[1] A.S. Pronikov, Parametric reliability of machines, M.: Publishing House of Moscow State Technical University, N.E. Bauman, 2002. 
[2] A.A. Ignatiev, V.V. Gorbunov, S.A. Ignatiev, Monitoring the process as an element of product quality management, Saratov: SSTU, 2009.

[3] V.A. Kudinov, Dynamics of machine tools, M .: Mashinostroenie, 1967. $360 \mathrm{p}$.

[4] A.A. Ignatiev, V.A. Karakozova, S.A. Ignatiev, Stochastic identification methods in the dynamics of machine tools, Saratov, SSTU, 2013.

[5] M.M. Arshansky, V.P. Shcherbakov, Vibration diagnostics and precision control on machine tools, M .: Mashinostroenie, 1989.

[6] M.P. Kozochkin, "The influence of the dynamic characteristics of machine tools on vibration during cutting," STIN, 2014, №2, pp. 4-9.

[7] A.A. Ignatiev, M.V. Vinogradov, V.V. Gorbunov et al., "Monitoring of machine tools and grinding processes in bearing production," Saratov: Saratov State Technical University, 2004.

[8] A.A. Ignatiev, V.A. Karakozova, S.A. Ignatiev, Transfer Functionand Margin of Stability in the Dynamic System of a Grinding Machine," RussianEngineeringResearch, 2015, Vol. 35, No. 2, pp. 123-125.

[9] B.M. Brzhozovsky, A.A. Ignatiev, V.A. Dobryakov, V.V. Martynov, Accuracy and reliability of automated machine tools: in 3 parts, Saratov: SPI, 1994, Part 2

[10] A.A. Ignatiev, E.M. Samoilova, Y.Sh. Shamsadova, "Assessment of the dynamic quality of machines using autocorrelation functions of vibroacoustic oscillations," News of universities, Volga region. Technical science, 2017, No. 2 (42), pp. 90-98.

[11] V.I. Popov, V.I. Loktev, Dynamics of machine tools, Kiev: Technique, 1975

[12] Z.H. Lin, D.C. Hodgson, "In-process measurement and analysis of the characteristics of the machine tool structures," Int. J. Mach. TooIsManufact, Vol. 28, No. 2, pp. 93-101, 1988.

[13] K.V. Egorov, Fundamentals of automatic regulation, M.: Energia, 1967, 648. 